\title{
Understanding the complex pathophysiology of idiopathic intracranial hypertension and the evolving role of venous sinus stenting: a comprehensive review of the literature
}

\author{
Nisha Giridharan, BS, ${ }^{1}$ Smruti K. Patel, MD, ${ }^{1}$ Amanda Ojugbeli, BS, ${ }^{1}$ Aria Nouri, MD, MSc, ${ }^{1}$ \\ Peyman Shirani, MD, ${ }^{1,2}$ Aaron W. Grossman, MD, PhD, ${ }^{1,2}$ Joseph Cheng, MD, MS, ${ }^{1}$ \\ Mario Zuccarello, MD, ${ }^{1}$ and Charles J. Prestigiacomo, MD ${ }^{1}$
}

Departments of ${ }^{1}$ Neurosurgery and ${ }^{2}$ Neurology, University of Cincinnati College of Medicine, Cincinnati, Ohio

\begin{abstract}
Idiopathic intracranial hypertension $(\mathrm{IIH})$ is a disease defined by elevated intracranial pressure without established etiology. Although there is now consensus on the definition of the disorder, its complex pathophysiology remains elusive. The most common clinical symptoms of IIH include headache and visual complaints. Many current theories regarding the etiology of IIH focus on increased secretion or decreased absorption of cerebrospinal fluid (CSF) and on cerebral venous outflow obstruction due to venous sinus stenosis. In addition, it has been postulated that obesity plays a role, given its prevalence in this population of patients. Several treatments, including optic nerve sheath fenestration, CSF diversion with ventriculoperitoneal or lumboperitoneal shunts, and more recently venous sinus stenting, have been described for medically refractory $\mathrm{IH}$. Despite the availability of these treatments, no guidelines or standard management algorithms exist for the treatment of this disorder. In this paper, the authors provide a review of the literature on $\mathrm{IIH}$, its clinical presentation, pathophysiology, and evidence supporting treatment strategies, with a specific focus on the role of venous sinus stenting. https://thejns.org/doi/abs/10.3171/2018.4.FOCUS18100
\end{abstract}

KEYWORDS idiopathic intracranial hypertension; benign intracranial hypertension; pseudotumor cerebri; venous sinus stenting; CSF diversion; optic nerve sheath fenestration

$\mathrm{I}$ DIOPATHIC intracranial hypertension (IIH), formerly known as pseudotumor cerebri, is a disorder characterized by elevated intracranial pressure (ICP) and remains a diagnosis of exclusion. ${ }^{16}$ IIH has an incidence of $0.5-2 / 100,000$ in the general population and a greater incidence among women of child-bearing age (12-20 per $100,000)$, typically presenting between 25 and 36 years of age. ${ }^{22}$ IIH is 10 times more common in women than in men, and obesity increases the risk of developing IIH approximately 20 -fold. ${ }^{16}$ The clinical diagnosis of IIH is based on a series of classic signs and symptoms in the absence of any intracranial findings; however, the etiology of IIH remains elusive and largely theoretical. Thus, many treatment options or recommendations are driven by these theories, including those related to abnormal cerebrospinal fluid (CSF) physiology, such as increased production and/or decreased absorption, and to pressure differentials within the venous sinus system. The present paper serves as an updated, comprehensive review of $\mathrm{IIH}$, including the descriptions of its clinical presentation, pathophysiology, treatment, and management strategies, with a special focus on venous sinus stenting (VSS).

\section{Clinical Presentation}

The clinical presentation of IIH varies among patients; however, most patients present with headaches and visual changes. The most commonly reported symptom of $\mathrm{IIH}$ is generalized headaches, reported by $75 \%-94 \%$ of patients. ${ }^{22}$ Headaches are severe upon awakening and are often described as pressure-like; they can be located in the frontal or retro-orbital regions or involve the whole head..$^{22}$

ABBREVIATIONS CSF = cerebrospinal fluid; ICP = intracranial pressure; IIH = idiopathic intracranial hypertension; LP = lumbar puncture; LPS = lumboperitoneal shunt; $\mathrm{MRV}=$ magnetic resonance venography; ONSF = optic nerve sheath fenestration; $R C T s=$ randomized controlled trials; VPS = ventriculoperitoneal shunt; VSS = venous sinus stenting.

SUBMITTED February 28, 2018. ACCEPTED April 23, 2018.

INCLUDE WHEN CITING DOI: 10.3171/2018.4.FOCUS18100. 
Patients also report migraine-type headaches that involve unilateral or bilateral facial throbbing, accompanied by nausea, photophobia, and/or phonophobia. ${ }^{22}$ Other frequently reported symptoms include neck pain, back pain, pulsatile tinnitus, and nausea/vomiting. Transient vision loss is the second most commonly reported symptom. Visual complaints may also encompass double vision, reduction in visual acuity, enlarging blind spot, and visual field deficits. ${ }^{16}$ In rare cases of fulminant IIH, severe vision loss can occur $<4$ weeks from the onset of symptoms, and surgical intervention is often required in such cases to prevent permanent blindness. ${ }^{22}$ Visual morbidity is the most severe complication of this disorder.

Elevated ICP is corroborated by the presence of papilledema in nearly all cases ( $95 \%$ of cases), although this is not a diagnostic requirement, and it is characterized by elevation of the optic disc, blurring of the optic disc margin, and obscuration of major vessels. ${ }^{22}$ Papilledema is associated with the above-described visual changes, resulting in permanent vision loss if treatment is delayed. Markey et al. ${ }^{22}$ reported that $1 \%-2 \%$ of IIH patients suffered permanent vision loss secondary to papilledema. Pulsatile tinnitus was reported in $52 \%-60 \%$ of patients, with the condition being bilateral in $66 \%$ of these cases. ${ }^{22}$ Due to the variability among patients, the modified Dandy criteria $^{16}$ and its modern adaptations are used to aid in diagnosing IIH (Table 1). Although these measures are supporting criteria for diagnosing IIH, the condition is considered a diagnosis of exclusion. Based on our literature review and current practice patterns, Fig. 1 provides a flow-chart algorithm for diagnostic workup of IIH.

\section{Pathophysiology}

Due to the unclear etiology of IIH, the pathophysiology of the disorder remains highly speculative. Given the constellation of clinical symptoms indicating a high ICP in the absence of hydrocephalus or ventriculomegaly, the vast majority of the IIH literature has focused on the tenets of increased CSF production, decreased CSF absorption, increased venous sinus pressure, and factors such as obesity that may influence these mechanisms (Fig. 2).

\section{Increased CSF Production}

Although the ependymal lining of the ventricles contributes to CSF production, the majority is produced by epithelial cells of the choroid plexus. Consequently, the choroid plexus has become a major focus of IIH research, and it has been theorized that abnormalities may result in increased CSF production. ${ }^{22}$ Indeed, tumors of the choroid plexus must be excluded when considering CSF hypersecretion in IIH.

\section{Decreased CSF Reabsorption}

The mechanisms that control CSF drainage are poorly understood, but it is largely accepted that CSF outflow

\section{TABLE 1. Original modified Dandy criteria and the criteria utilized in the Idiopathic Intracranial Hypertension Treatment Trial}

A: Modified Dandy Criteria for Idiopathic Intracranial Hypertension*

\footnotetext{
1. Signs and symptoms of increased ICP (headaches, nausea, vomiting, transient visual obscurations, or papilledema)

2. Absence of localized findings in neurological examination (except for false localizing signs such as abducens nerve palsy)

3. Normal CT/MRI findings without evidence of hydrocephalus or mass lesion

4. CSF opening pressure $>25 \mathrm{~cm} \mathrm{H}_{2} \mathrm{O}$ with normal CSF cytological and chemical findings

5. No other causes of elevated intracranial pressure identified
}

B: Idiopathic Intracranial Hypertension Treatment Trial-Modified Dandy Criteria†

\section{Signs and symptoms of increased ICP}

\section{Absence of localizing findings on neurologic examination}

4. Absence of deformity, displacement, or obstruction of the ventricular system and otherwise normal neurodiagnostic studies, except for evidence of increased CSF pressure ( $>200 \mathrm{~mm} \mathrm{H}_{2} \mathrm{O}$ ); abnormal neuroimaging except for empty sella turcica, optic nerve sheath with filled-out CSF spaces, and smooth-walled non-flow-related venous sinus stenosis or collapse should lead to another diagnosis

4. Awake and alert

5. No other cause of increased ICP

If the CSF opening pressure was $200-250 \mathrm{~mm} \mathrm{H}_{2} \mathrm{O}$, at least one of the following was also required:

- Pulse synchronous tinnitus

- CN VI palsy

- Frisen grade II papilledema

- Echography for drusen-negative and no other disc anomalies mimicking disc edema present

- MRV with lateral sinus collapse/stenosis preferable using auto-triggered elliptic centric-ordered technique

- Partially empty sella on coronal or sagittal views and optic nerve sheaths with filled-out CSF spaces next to globe on T2-weighted axial scans

$\mathrm{CN}=$ cranial nerve.

* Modified Dandy Criteria initially proposed in 1985. Adapted from Friedman DI, Jacobson DM. Diagnostic criteria for idiopathic intracranial hypertension. Neurology 59(10):492-1495, 2002

$\dagger$ Idiopathic Intracranial Hypertension Treatment Trial updated version of the Modified Dandy criteria with further revisions allowing for the "probable" diagnosis of IIH syndrome in certain circumstances. Adapted from Wall M, Corbett JJ. Revised diagnostic criteria for the pseudotumor cerebri syndrome in adults and children. Neurology 83(2):198-200, 2014. 


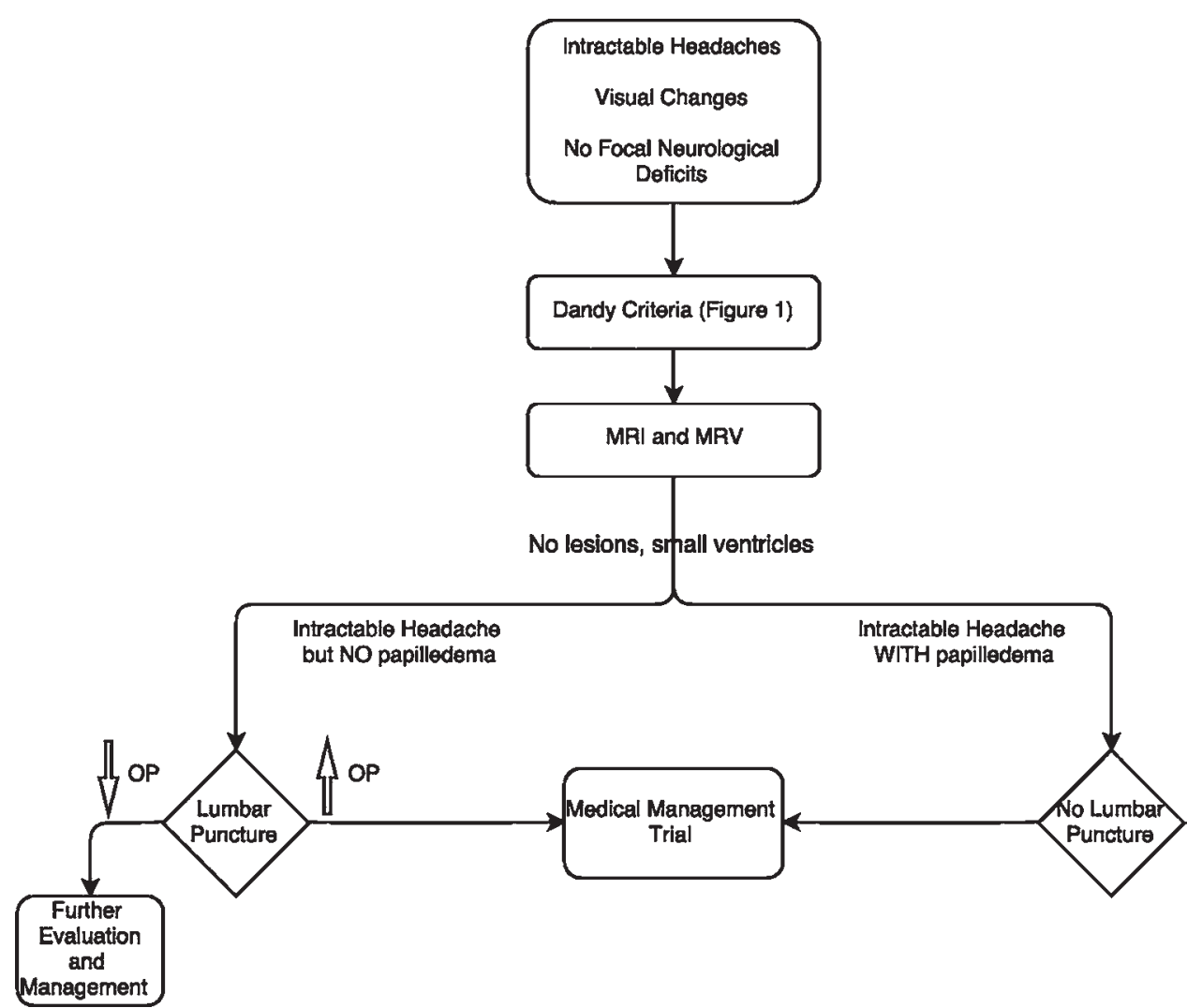

FIG. 1. Proposed flow-chart algorithm for initial diagnostic workup of IIH based on the tenets of the modified Dandy criteria. We suggest that for patients who meet clinical criteria, imaging workup with MRI and MRV be completed; if negative, then further workup with lumbar puncture is performed based on the presence or absence of papilledema. All patients who are found to meet criteria with elevated ICP should undergo a medical management trial. For those patients with clinical symptoms suggestive of IIH without an elevated opening pressure, further evaluation and management is warranted. $O P=$ opening pressure

through arachnoid granulations into the cortical venous sinuses plays a critical role. ${ }^{8}$ Cerebral venous outflow obstruction remains a point of interest in the pathophysiology underlying $\mathrm{IIH}$, and obstruction due to meningitis and hemorrhage must be excluded as potential causes. Absorption of CSF across the arachnoid villi is proportional to the pressure gradient between the venous sinuses and subarachnoid space and inversely proportional to flow resistance. ${ }^{25}$ Thus, it is suggested that an increase in venous pressure results in an increase in ICP due to decreased CSF absorption related to the loss in the CSF-venous pressure gradient. ${ }^{22}$ The presence of venous sinus stenosis in a subset of patients with IIH has provided some support for this potential mechanism (Fig. 3). It is uncertain whether the stenosis results in venous hypertension, or whether the stenosis is a consequence of external compression of the vessel. ${ }^{11}$ However, the results of VSS as an intervention for IIH have been promising, ${ }^{4}$ regardless of the cause of the stenosis.

\section{Management of IIH \\ Medical Therapy}

The first-line treatment for IIH is medical management and weight loss. Randomized controlled trials (RCTs) have shown modest improvement in visual acuity with the carbonic anhydrase inhibitor acetazolamide compared to placebo (class IB evidence). ${ }^{43}$ The IIH Treatment Trial showed the following statistically significant effects of acetazolamide: 1) improved visual field function, 2) decrease in papilledema grade, 3) improved quality-of-life measures, and 4) decreased CSF pressure when compared to placebo. ${ }^{44}$ While no patients suffered permanent morbidity, those in the acetazolamide group did experience more adverse events, including electrolyte disturbances, nephrolithiasis, paresthesias, dysgeusia, nausea/vomiting, diarrhea, and fatigue. ${ }^{42}$ For patients who continue to remain symptomatic despite maximal medical therapy or those patients who do not tolerate acetazolamide, several surgical options exist including CSF diversion, optic nerve sheath fenestration (ONSF), bariatric surgery, and VSS. ${ }^{28}$ However, RCTs are still unavailable for these surgical options, making it difficult to determine best practices in cases of medically refractory IIH.

\section{Optic Nerve Sheath Fenestration}

Optic nerve sheath fenestration has been the recommended treatment for patients with medically refractory IIH who present with severe vision loss and minimal headache symptoms. ${ }^{15}$ The procedure involves creating slits in the edematous optic nerve sheath and has been associated with demonstrable postoperative improvement 


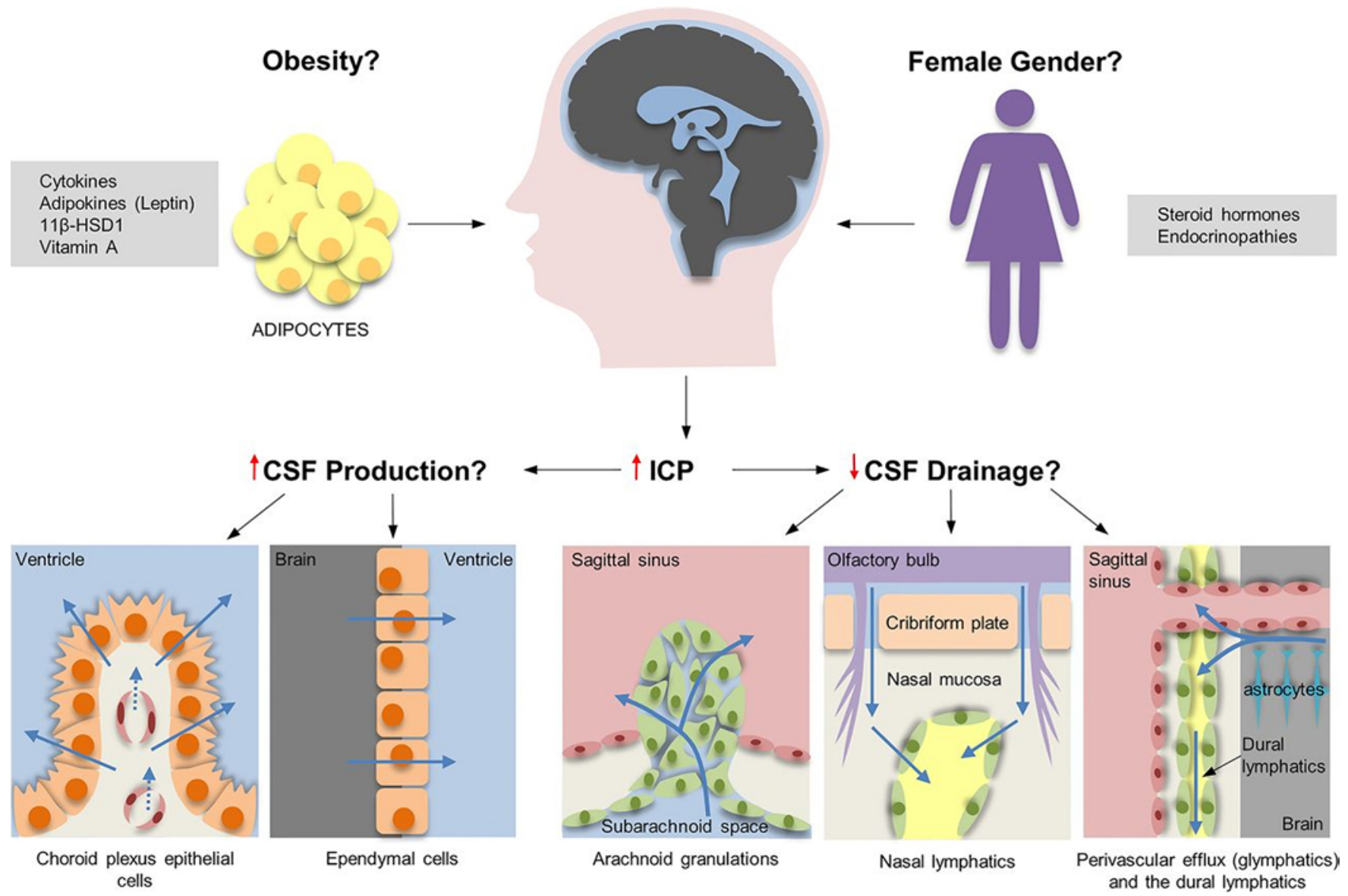

FIG. 2. Schematic illustration of the possible pathophysiological mechanisms underlying IIH. 1) Increased CSF production: CSF, produced by the epithelial cells of the choroid plexus and ependymal cells lining the ventricular system, can be hypersecreted, causing elevation of ICP. 2) Decreased CSF drainage: CSF is thought to primarily drain into the subarachnoid space via the arachnoid granulations into the superior sagittal sinus. In addition, there is evidence that CSF also drains through the nasal lymphatic system as well as through the glymphatic pathway (bulk flow of CSF along perivascular routes). In addition, obesity and female sex are known associated predisposing risk factors for the development of IIH. While the role of obesity remains largely unclear, researchers have proposed an inflammatory etiology. A number of studies have suggested that the use of steroid hormones (and steroid withdrawal) as well as in the setting of endocrinopathies may contributed to the development of intracranial hypertension. However, the individual use of hormones or hormonal contraception in the setting of IIH has been largely unsubstantiated. Reprinted (with modification of the legend) from Mollan SP, Ali F, Hassan-Smith G, et al: Evolving evidence in adult idiopathic intracranial hypertension: pathophysiology and management. J Neurol Neurosurg Psychiatry 2016;87: 982-992, 2016. CC BY 4.0 (https://creativecommons.org/licenses/by/4.0/).

in patients' visual acuity and visual fields (Fig. 4)..$^{13}$ The mechanism by which this procedure provides symptomatic relief for these patients is uncertain. One theory is that ONSF induces fibrosis, blocking the CSF flow between the subarachnoid space around the optic nerve and the intracranial subarachnoid space. ${ }^{6}$ Another theory is that ONSF creates a dural fistula, which allows CSF drainage, thus relieving intracranial hypertension.

In a meta-analysis of data obtained in IIH patients undergoing ONSF, Satti et al. ${ }^{37}$ reviewed 18 studies including 712 patients (1153 optic nerves). Almost all of the patients presented with reduced visual acuity $(92 \%)$ or visual field changes $(86 \%)$ and headache $(60 \%)$. The study reported improved visual acuity in 59\%, $95 \%$ had improvement or stable visual acuity, visual fields improved in $68 \%$, headaches improved in $44 \%$, and papilledema improved or resolved in $80 \% .37$
Major complications of the procedure occurred in 1.5\% of patients and included orbital or retrobulbar hematomas, orbital cellulitis, traumatic optic neuropathy, and strabismus. ${ }^{37}$ Minor complications occurred in $16.4 \%$ of patients and included diplopia, pupillary dysfunction, anisocoria, corneal dellen (localized thinning or drying of the cornea), and late failure. Overall, the complication rate was high at $18 \%$, with $15 \%$ of patients requiring additional procedures. By 6 to 12 months, the probability of failure was 0.16 by life table analysis, which doubled by 3 to 5 years $(0.35){ }^{39}$

\section{CSF Flow Diversion}

Given that increased CSF production is one possible mechanism for the pathophysiology of IIH, serial lumbar punctures can be performed in IIH patients to rapidly relieve intracranial hypertension and decrease ICP. Howev- 
N. Giridharan et al.
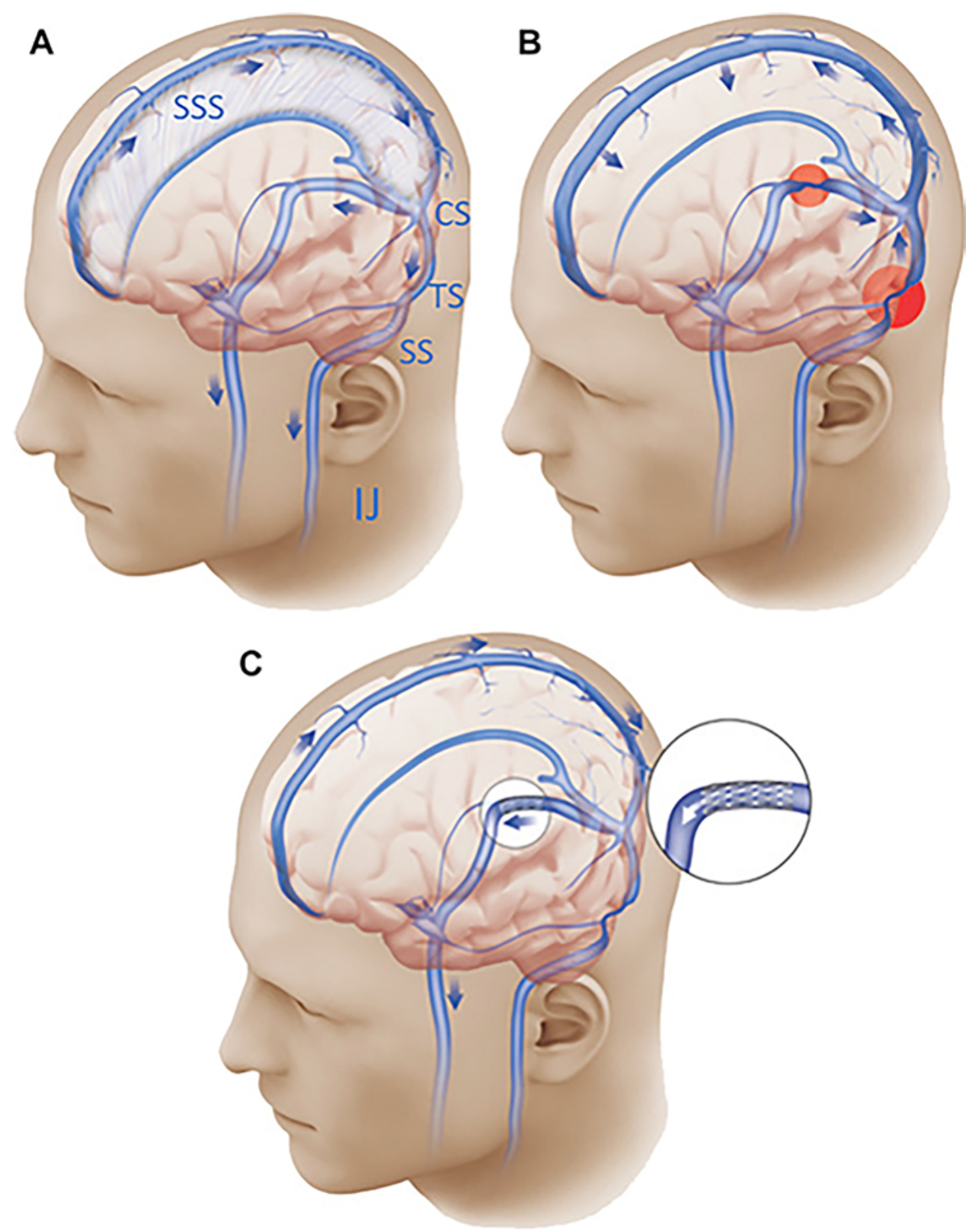

FIG. 3. A: Representative illustration demonstrating the normal flow of intracranial venous drainage from the superior sagittal sinus (SSS) to the confluence of the sinuses (CS) into the transverse sinuses (TS) and through the sigmoid sinus (SS) into the internal jugular vein (IJ). B: Illustration demonstrating bilateral transverse/sigmoid venous sinus stenosis, which results in an increased transstenotic gradient (differential pressures between the distal sigmoid sinus and the proximal transverse sinus segments) and in turn leads to venous outflow obstruction and venous hypertension, thus leading to decreased CSF absorption, elevated ICP and further venous sinus compression, and an even higher transstenotic gradient. C: Unilateral stenting of region of stenosis, which in theory restores the normal venous outflow, thus normalizing the transstenotic gradient and ICP. Figure reprinted from Neurol Clin 35(7), Dinkin MJ, Patsalides A, Venous sinus stenting for idiopathic intracranial hypertension: Where are we now? 59-81, copyright 2017, with permission from Elsevier.

er, patients who achieve the most benefit from intermittent high-volume lumbar puncture (LP) are unlikely to utilize this as a durable, long-term treatment. More often, highvolume LP is used as a temporizing measure until a more definitive procedure is performed. The most common definitive surgical treatment for IIH is placement of a ventriculoperitoneal (VPS) or lumboperitoneal shunt (LPS). A large meta-analysis comprising 17 studies including 435 patients who underwent CSF diversion for treatment of IIH showed good success in relieving symptoms of headache and signs of papilledema. ${ }^{37}$ The authors reported that $86 \%$ of patients who presented with headache experienced improvement in their headache with shunt placement. Papilledema improved in $70 \%$ of patients. Changes in visual acuity showed less improvement than the other symptoms.

Major complications were seen in $7.6 \%$ of patients, 


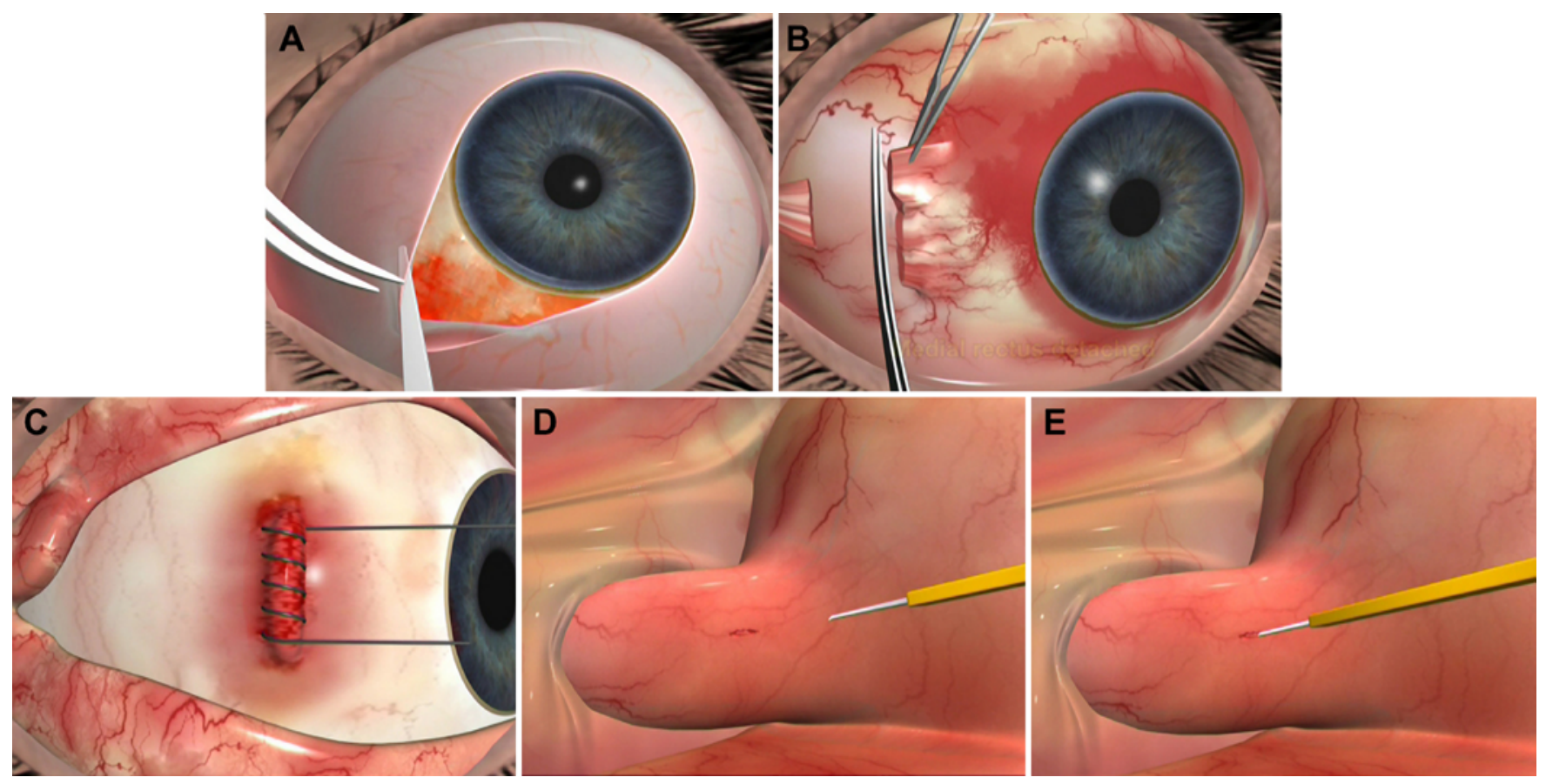

FIG. 4. Illustration demonstrating the basic steps of an optic nerve sheath fenestration procedure. A: A $270^{\circ}$ peritomy, or incision of the conjunctiva and subconjunctiva about the circumference of the cornea, is performed at the superior, medial, and inferior limbus. B: The medial rectus is hooked and imbricated with suture then cut, leaving behind a stump. C: A baseball traction suture is passed from one end of the cut medial rectus muscle stump and out through the other multiple times. D: The optic nerve is identified by following the long ciliary neurovascular bundle posteriorly. A microvitreoretinal blade is used to create a 2-mm longitudinal incision in an avascular portion of the dura. $\mathrm{E}$ : The fenestration is enlarged until it is about 4-6 mm long, and a nerve hook is placed under the subarachnoid space to lyse any adhesions. Reprinted from Agarwal A, Jacob S. Optic nerve sheath fenestration treats idiopathic intracranial hypertension. Ocular Surgery News U.S. Edition. December 25, 2012. Reprinted with permission from SLACK Incorporated.

including shunt infection, subdural hematoma, tonsillar herniation, and CSF fistula. ${ }^{37}$ About one-third of patients experienced a minor complication (143/435), ranging from abdominal pain to shunt disconnection/malposition to CSF leak and low-pressure headache. Overall, the complication rate was high, with $43 \%$ of patients requiring additional procedures, such as shunt revision for multiple indications. ${ }^{27}$ Whereas studies have shown symptomatic improvement immediately after CSF diversion surgery, long-term success has been less convincing. McGirt et al. ${ }^{26}$ found that $95 \%$ of patients had improvement in their headaches 1 month following VPS/LPS placement. Headaches recurred for $19 \%$ by 1 year and nearly half $(48 \%)$ by 36 months after the procedure. ${ }^{26}$ Sinclair et al. ${ }^{38}$ reviewed 53 cases over a 10 -year period, with $96 \%$ of patients presenting initially with headache due to increased ICP. ${ }^{38}$ At 6 months' follow-up after shunt placement, there was a statistically significant reduction in the number of patients complaining of high-pressure headaches, and only $63 \%$ were symptomatic. However, this increased to $77 \%$ at 1 year and $79 \%$ at 2 years.

\section{Venous Sinus Stenting}

Stenosis of the dural venous sinuses is common among patients with IIH. Elder et al. ${ }^{11}$ reported incidences of transverse sinus stenosis in IIH to range from $10 \%$ to $90 \%$, compared to the $6.8 \%$ incidence among the gener- al population. Farb et al. ${ }^{12}$ observed that $93 \%$ of patients had bilateral venous sinus stenosis on magnetic resonance venography (MRV) compared to 7\% of controls. Venous outflow obstruction was proposed even in the earliest description of IIH. In the first few decades of the 1900s, there were multiple reports of IIH presenting in conjunction with chronic otitis media and mastoiditis, with a suspected link between IIH and venous outflow obstruction. ${ }^{30}$ However, the introduction of antibiotics and their use in the treatment of otitis media and mastoiditis resulted in a decreased incidence of reported IIH cases, and ultimately led many to believe that venous outflow obstruction was not the source of IIH.

It was not until the end of the 20th century, with advances in imaging that demonstrated morphological abnormalities, that sinus obstruction again became the focus of IIH. ${ }^{30}$ With this link established, balloon angioplasty of venous sinus stenosis in patients with IIH has been attempted. However, the results have been disappointing, with patients experiencing a short-term relief of symptoms and a high rate of recurrence. ${ }^{30}$ The failure of this therapy was attributed to not addressing the underlying cause of the venous sinus obstruction. While there is some disagreement in the literature about whether venous sinus stenosis results from an intrinsic or extrinsic process, endovascular stenting has demonstrated efficacy in medically refractory patients. 


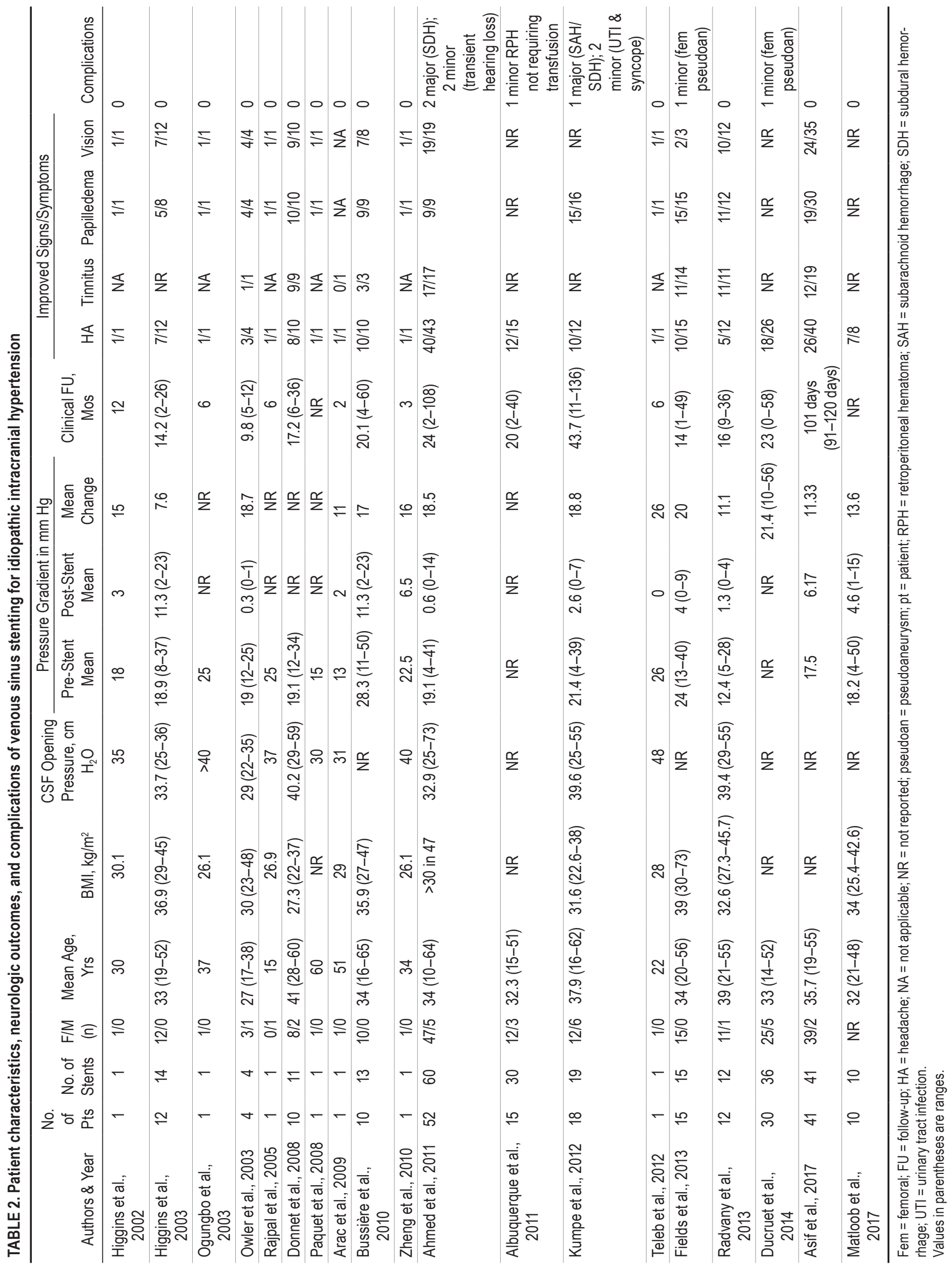


TABLE 3. Radiologic follow-up and stent-related outcomes of venous sinus stenting for idiopathic intracranial hypertension

\begin{tabular}{|c|c|c|c|c|c|c|c|}
\hline Authors \& Year & $\begin{array}{l}\text { No. of } \\
\text { Pts }\end{array}$ & $\begin{array}{l}\text { No. of } \\
\text { Stents }\end{array}$ & $\begin{array}{c}\text { Avg } \\
\text { Radiographic FU }\end{array}$ & $\begin{array}{l}\text { No. of In-Stent } \\
\text { Stenoses }\end{array}$ & Subsequent Treatment & $\begin{array}{l}\text { No. of Out-of-Stent } \\
\text { Stenoses }\end{array}$ & $\begin{array}{l}\text { Subsequent } \\
\text { Treatment }\end{array}$ \\
\hline Higgins et al., 2002 & 1 & 1 & $1 \mathrm{mo}$ & 0 & 0 & 0 & 0 \\
\hline Higgins et al., 2003 & 12 & 14 & NR & 2 & Thrombolytic therapy & NR & NR \\
\hline Ogungbo et al., 2003 & 1 & 1 & $12 \mathrm{mos}$ & 0 & 0 & 0 & 0 \\
\hline Owler et al., 2003 & 4 & 4 & $3 \mathrm{mos}$ & 0 & 0 & 1 & 0 \\
\hline Rajpal et al., 2005 & 1 & 1 & $6 \mathrm{mos}$ & 0 & 0 & 0 & 0 \\
\hline Donnet et al., 2008 & 10 & 11 & $6 \mathrm{mos}$ & 0 & 0 & 0 & 0 \\
\hline Paquet et al., 2008 & 1 & 1 & NR & NR & NR & NR & NR \\
\hline Arac et al., 2009 & 1 & 1 & $2 \mathrm{mos}$ & 0 & 0 & 0 & 0 \\
\hline Bussière et al., 2010 & 10 & 13 & NR & NR & NR & NR & NR \\
\hline Zheng et al., 2010 & 1 & 1 & NR & NR & NR & NR & NR \\
\hline Ahmed et al., 2011 & 52 & 60 & NR & 0 & 0 & 6 & Restenting in 6 pts \\
\hline Albuquerque et al., 2011 & 15 & 30 & $12.5 \mathrm{mos}$ & 0 & 0 & 1 & Restenting \\
\hline Kumpe et al., 2012 & 18 & 19 & $25.3 \mathrm{mos}$ & 0 & 0 & 4 & Restenting in $1 \mathrm{pt}$ \\
\hline Teleb et al., 2012 & 1 & 1 & $12 \mathrm{mos}$ & 0 & 0 & 0 & 0 \\
\hline Fields et al., 2013 & 15 & 15 & $9 \mathrm{mos}$ & 0 & 0 & 0 & 0 \\
\hline Radvany et al., 2013 & 12 & 12 & NA & 0 & 0 & 2 & $\begin{array}{r}\text { Restenting in } 2 \text { pts \& } \\
1 \text { pt requiring VPS }\end{array}$ \\
\hline Ducruet et al., 2014 & 30 & 36 & $22 \mathrm{mos}$ & 4 & 0 & 5 & 0 \\
\hline Asif et al., 2017 & 41 & 41 & $\begin{array}{r}101 \text { days (range } \\
91-120 \text { days) }\end{array}$ & 2 & $\begin{array}{l}\text { Restenting in } 1 \mathrm{pt} \text {, throm- } \\
\text { bolytic therapy in } 1 \mathrm{pt}\end{array}$ & 3 & Restenting in 3 pts \\
\hline Matloob et al., 2017 & 10 & 10 & 3 & NR & NR & NR & NR \\
\hline
\end{tabular}

Avg = average.

\section{Methods}

A structured literature search was conducted to identify studies reporting outcomes for venous sinus stenting (VSS) used to treat idiopathic intracranial hypertension (IIT). The electronic database MEDLINE (PubMed) was searched using relevant combinations of appropriate keywords including "idiopathic intracranial hypertension," "pseudotumor cerebri," "benign intracranial hypertension," "venous sinus," and "stent." English-language studies published before February 2018 were screened based on titles and abstracts to determine inclusion. Additional studies were found by reviewing the reference lists of the identified publications. All included studies are displayed in Tables 2 and 3.

\section{Literature Review}

The first successful report of the use of dural VSS for refractory IIH was by Higgins et al. ${ }^{18}$ In a patient with partial bilateral transverse sinus stenosis, the authors showed a significant reduction in the pressure gradient across the stenotic segments after placement of a self-expanding stent. The patient reported improvement in headaches, and the authors noted resolution of the patient's papilledema. Two case reports of patients with IIH treated with VSS followed the work of Higgins et al., demonstrating a reduction in the pressure gradient across the stenotic area of the venous sinus after deployment of a stent. ${ }^{30}$ More recent case series have replicated this initial early success.

In all patients with IIH considered for VSS, preproce- dural testing is used to identify appropriate candidates; initial screening is done with MRV (Fig. 5A). For those with evidence of sinus stenosis, further testing can include digital subtraction venography and manometry under monitored anesthesia care with a plan for stenting under general anesthesia on another date if a significant pressure gradient is measured across the stenosis. ${ }^{20}$ Other options include performing the digital subtraction venography and manometry under general anesthesia with intent to treat at that time if a significant pressure gradient is recorded. Most authors reported stenting in patients with a pressure gradient $\geq 10 \mathrm{~mm} \mathrm{Hg}$ (Fig. $5 \mathrm{~B}$ and C). ${ }^{20}$ Unilateral VSS was performed more often even if the patient had evidence of bilateral stenosis. Commonly, the stent was placed on the side with the greater degree of stenosis or in the dominant transverse/sigmoid sinus. ${ }^{20}$ In a meta-analysis of 143 IIH patients undergoing VSS, 96\% received unilateral stents, and $69 \%$ of the stents were placed in the right transverse/sigmoid sinus. ${ }^{34}$

Large-scale studies have analyzed the efficacy and safety of VSS. Starke et al. ${ }^{40}$ reviewed 17 studies from 2002 to 2014 with 185 patients receiving VSS (Tables 2 and 3 in their report). ${ }^{1,3-5,7,9,10,14,17,18,21,24,29,31,32,35,36,41,45}$ The locations of stent placement were not included. The authors reported that $78.3 \%$ of patients presenting with headache experienced some relief or complete resolution following stent placement ${ }^{40}$ For patients with visual changes, $86.5 \%$ had improvement or restoration of their normal vision after the procedure, while papilledema improved or resolved in all but 5 patients (94.4\%). Tinnitus improved in $92.9 \%$ 
of patients. ${ }^{40}$ The mean pressure gradient across the stenotic venous segments decreased from $20.1 \mathrm{~mm} \mathrm{Hg}$ prior to stenting to $4.4 \mathrm{~mm} \mathrm{Hg}$ afterwards. ${ }^{40}$ A recent case series by Matloob et al. ${ }^{24}$ was the first study of patients to undergo VSS with real-time intraparenchymal pressure monitoring. The assumption in previous studies was that a resolution in the pressure gradient across the stenosis was a surrogate marker for a decrease in ICP. Matloob et al. ${ }^{24}$ demonstrated that resolution of the venous sinus stenosis and pressure gradient with stenting also correlated with an immediate reduction in ICP that was sustained at 24 hours in 9 of the 10 patients.

In the aforementioned studies, 3 patients experienced major complications (subdural, subarachnoid, and intraparenchymal hemorrhages). ${ }^{40}$ Seven had minor complications related to vascular access during the angiography procedure, which included a retroperitoneal hematoma and femoral pseudoaneurysms. Four patients with complications required another procedure: 2 craniotomies, an external ventricular drain placement, and 1 femoral artery stent placement for pseudoaneurysm. ${ }^{40}$ Overall, the reported complication rate was low at 5.4\% (10/185). Follow-up imaging was obtained at an average of 15 months postoperatively. Stenosis at the site of the stent was seen in 6 patients, with 1 patient requiring thrombolytic therapy. However, adjacent stenosis was found in 19 patients, with 10 requiring subsequent restenting or shunt placement $(6 \%$ of the overall VSS population). ${ }^{40}$ Others have reported re- vision rates with VSS as high as 13\%. ${ }^{2}$ While additional studies have shown lower complication rates with VSS than with shunting, ${ }^{5}$ the clinical outcomes are not easily comparable, and a randomized, prospective study would provide the greatest evidence to determine the optimal treatment option.

\section{Discussion}

Since Quincke's first description in the late $1800 \mathrm{~s},{ }^{8}$ our understanding of IIH as a disorder has evolved. In 1937, Walter Dandy described specific criteria for the disorder, which would later be called pseudotumor cerebri and subsequently idiopathic intracranial hypertension. ${ }^{8}$ Despite our increased knowledge regarding IIH, our understanding of the pathophysiology is still uncertain. Many theories have been proposed, including hypersecretion of CSF at the choroid plexus, decreased CSF absorption at the arachnoid granulations, and increased venous sinus pressure due to venous sinus stenosis.

Optic nerve sheath fenestration has traditionally been recommended in patients with predominant symptoms of vision loss without headache, yet emerging data on VSS have shown comparably good outcomes for improvement in visual acuity and visual fields in large meta-analyses (class IIA evidence). ${ }^{37}$ However, unlike studies involving ONSF, studies of stent placement rarely provide objective measures of pre- and postprocedure visual acuity and vi-

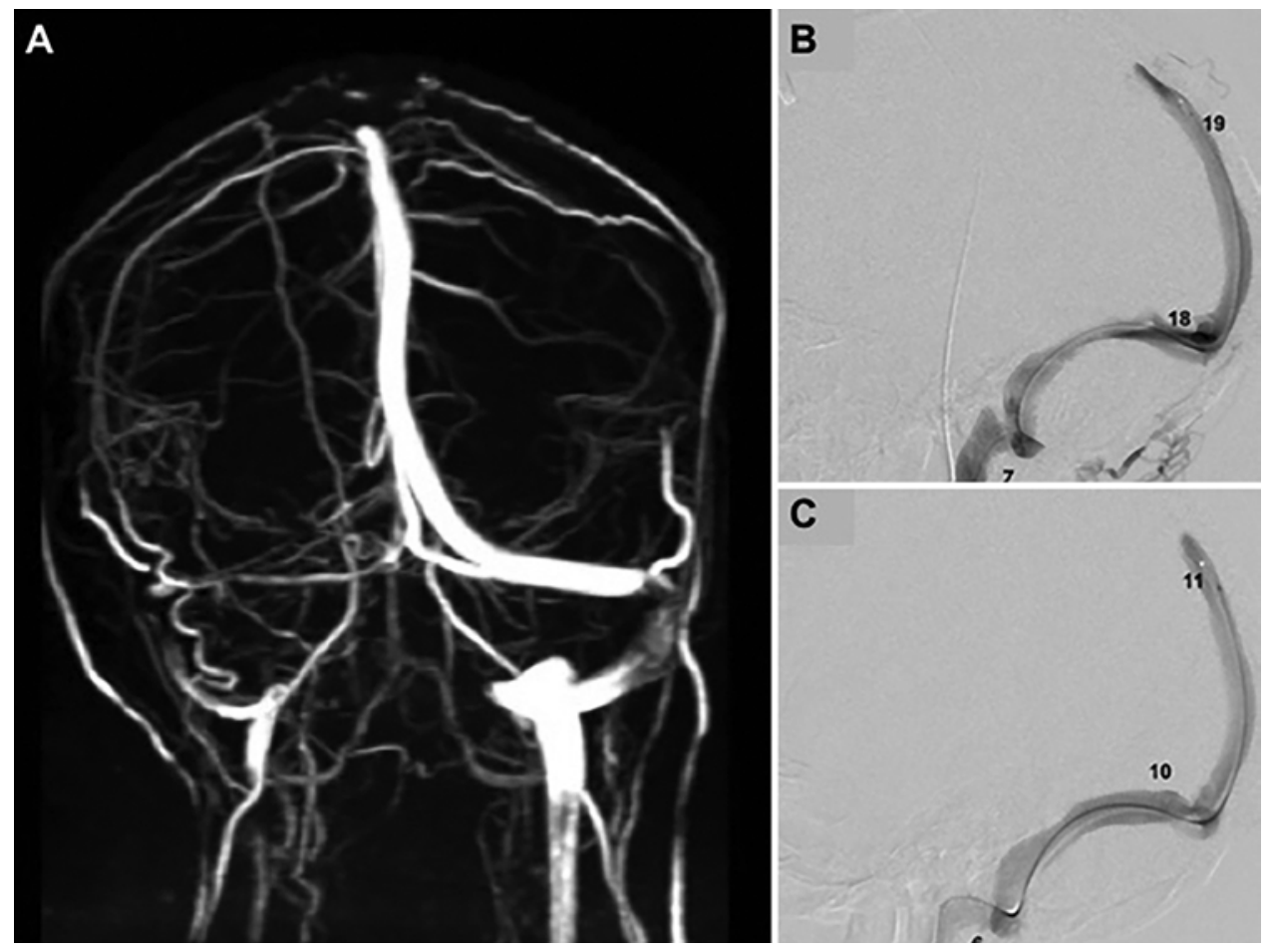

FIG. 5. A: An MR venography image (posterior view) demonstrating significant left transverse sinus stenosis. Reprinted (with modification of the description) from Mollan SP, Ali F, Hassan-Smith G, et al: Evolving evidence in adult idiopathic intracranial hypertension: pathophysiology and management. J Neurol Neurosurg Psychiatry 87:982-992, 2016. CC BY 4.0 license (https:// creativecommons.org/licenses/by/4.0/). B: Transverse sinus stenosis with a large pressure gradient prior to stent placement. C: Resolution of sinus stenosis and improvement of pressure gradient after stent insertion. The numbers represent pressure $(\mathrm{mm} \mathrm{Hg})$ measured in each location. Panels $B$ and $\mathrm{C}$ reprinted with permission from Asif $\mathrm{H}, \mathrm{Craven} \mathrm{CL}$, Siddiqui AH, Shah SN, Matloob SA, Thorne L, et al: Idiopathic intracranial hypertension: 120-day clinical, radiological, and manometric outcomes after stent insertion into the dural venous sinus. J Neurosurg [epub ahead of print October 6, 2017. DOI: 10.3171/2017.4.JNS162871]. 
sual field testing. Thus, reliable conclusions about the true efficacy of VSS in improving visual function are difficult to make. Based on our review of the existing evidence, we recommend that ONSF be considered in patients whose symptoms are refractory to medical therapy, with the primary symptoms of vision loss, minimal to no headache, and no radiographic evidence of venous sinus stenosis, and particularly in patients with rapidly progressive vision loss requiring urgent surgery.

Current review of the literature shows that CSF diversion is the most common surgical treatment option for
IIH. Historically, CSF diversion has very good short-term success in alleviating headache and signs of papilledema (class IIA evidence); however, close to half of patients experience recurrence of their headaches by 36 months after the surgery. ${ }^{26}$ This high rate of failure is accompanied by an equally high rate of revision surgeries; many patients require several additional procedures to address complications such as shunt obstruction, disconnection, and infection. In a cost analysis, Ahmed et al. ${ }^{2}$ found that the overall cost of CSF shunting was much greater than that of stenting due to the rate of revision. There was no statistically signifi-

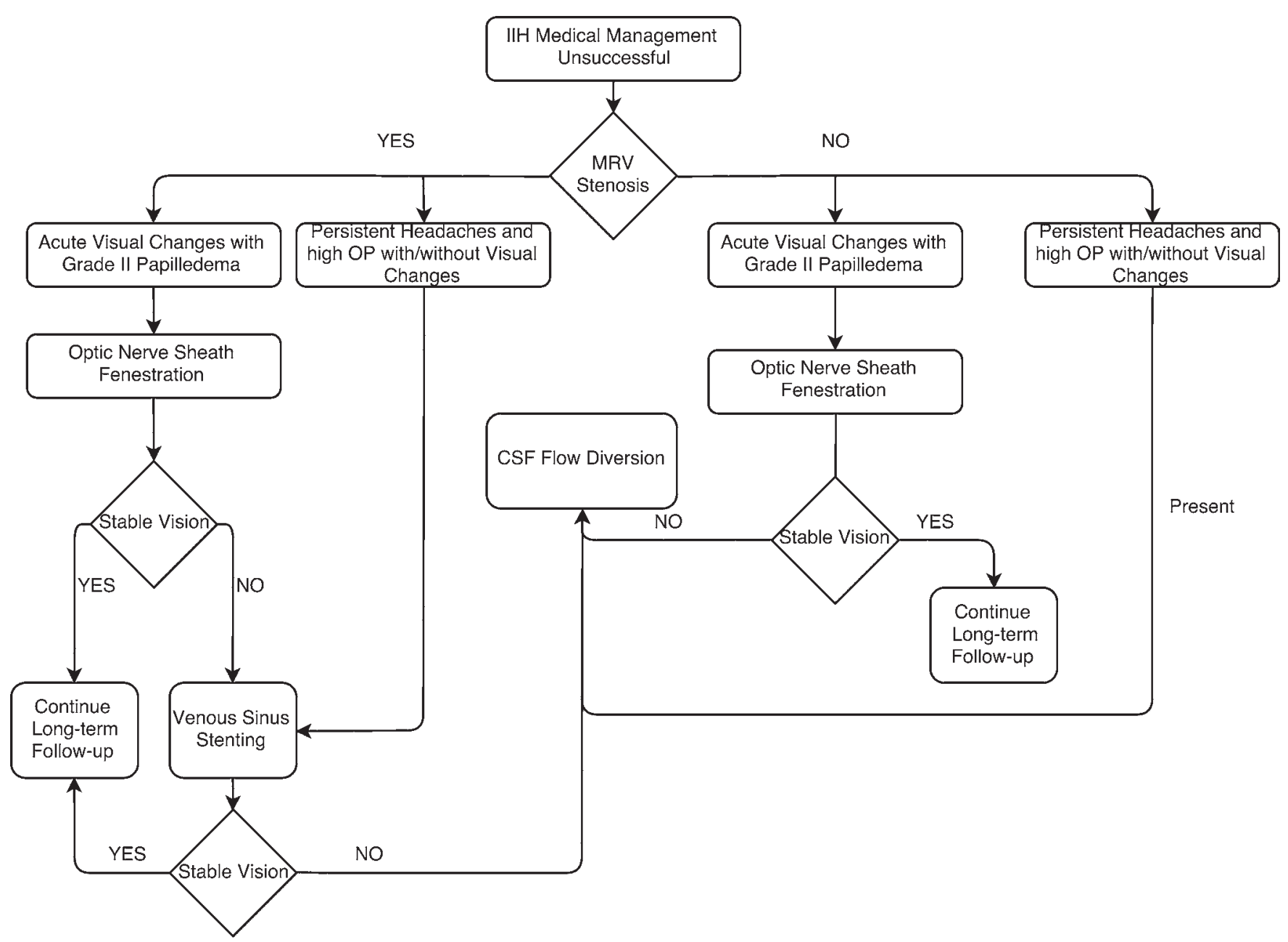

FIG. 6. Proposed flow-chart algorithm for treatment and management of medically refractory IIH. For patients with IIH who do not tolerate or experience persistence of symptoms despite maximal medical management, several interventions exist. As part of the proposed initial workup for IIH (Fig. 1), patients should have an MRV to assess for venous sinus stenosis. For those patients who have evidence of venous sinus stenosis on MRV, persistent headaches, and elevated opening pressures objectively measured by lumbar puncture, with or without visual changes, the provider should consider consulting an endovascular expert to discuss the option of venous sinus stenting (VSS) as a treatment. If after VSS, the patient experiences resolution of headache and stable vision, then long-term follow-up can be continued. If the patient's vision continues to deteriorate, then CSF diversion should be considered as the next therapeutic option. For those patients who have no evidence of venous sinus stenosis on MRV and persistent headaches and high opening pressure, with or without vision changes, we recommend CSF flow diversion as the most appropriate surgical option. In all patients with acute visual changes and grade II papilledema, we recommend proceeding with optic nerve sheath fenestration (ONSF). If the patient's visual complaints remain stable, continued long-term follow-up is recommend. If visual complaints persist after ONSF for patients with evidence of venous sinus stenosis on MRV, practitioners should consider a trial of VSS. If VSS is selected as the therapeutic option and fails to control visual changes, CSF flow diversion can alternatively be offered to the patient for symptomatic relief. For those patients with acute visual changes whose symptoms are not relieved by ONSF and who have no evidence of venous sinus stenosis on MRV, we recommend CSF flow diversion. 
cant difference in the initial costs of CSF shunting and venous stenting, with an average cost of $\$ 15,797$ for shunting and $\$ 13,863$ for stenting. ${ }^{2}$ The per procedure cost for additional procedures (additional stent placement procedures or shunt revision surgery) also did not differ significantly, but the shunt-treated group had a much higher rate of revisions (55\% compared to $13 \%$ for the stent-treated group). Overall, the cost per 100 cases for CSF diversion was almost twice the cost for VSS. ${ }^{2}$

Venous sinus stenting is a newer treatment option with a growing body of literature supporting its use in patients with medically refractory IIH and the presence of venous sinus stenosis. MRV is an excellent noninvasive imaging modality that may screen patients for venous sinus stenosis, with high sensitivity and high negative predictive value for this condition. ${ }^{19}$ Patients demonstrating bilateral transverse/sigmoid sinus stenosis may be appropriate candidates for this procedure as well as patients with evidence of unilateral transverse/sigmoid sinus stenosis and hypoplasia/aplasia of the contralateral side. ${ }^{37}$ Further workup for the procedure should include manometry for detection of an elevated proximal-to-distal pressure gradient across the stenosis. ${ }^{16}$ Currently, MRV and manometry are not routine steps in the workup for patients with medically refractory IIH, but given the promising results of the procedure, the authors recommend that they should be utilized during the diagnostic workup.

Several retrospective studies have shown that most patients with IIH presenting with headache, visual changes, or tinnitus achieved some symptomatic relief or even complete resolution of symptoms after venous stent placement. Additionally, the rates of restenosis on follow-up imaging at 15 months were about $14 \%$, with the literature reporting revision rates between $6 \%$ and $13 \% .^{2,40}$ In-stent stenosis and occlusion is thought to arise from in-stent thrombosis; short-term dual antiplatelet therapy has been shown to reduce the incidence of in-stent thrombosis ${ }^{20}$ and should be considered as part of the periprocedural protocol. The treatment of stent-adjacent stenosis is more controversial, as the pathophysiology remains unclear. This out-of-stent stenosis could represent increased pressure on the sinus, again calling into question whether venous sinus stenosis is the result or cause of increased ICP. ${ }^{40}$

Complications of VSS were rare and mostly found to be related to the angiography procedure itself, such as femoral pseudoaneurysms. ${ }^{40}$ Additionally, complications of ipsilateral frontal headache have been reported after the procedure, ${ }^{1}$ thought to be secondary to dural stretching from the stent placement. Neuropathic pain medications such as gabapentin can be used to treat patients with this complication. ${ }^{24}$ More serious concerns include intracranial hemorrhage, but this complication was only reported in 3 patients, all of whom made a complete recovery with emergent procedures. ${ }^{40}$

Studies examining the long-term outcomes of patients with VSS as well as the optimal candidates for stenting are required to formulate specific practice guidelines. RCTs comparing the outcomes of each of the surgical options are lacking. In addition, debate still exists regarding VSS and its risk/benefit ratio due to the paucity of the literature on this treatment. ${ }^{33}$ Current data suggest high efficacy and safety of stent placement and lower repeat-procedure rates compared with ONSF and CSF shunting (class IIA evidence). ${ }^{37}$ We recommend that MRV screening be considered to assess for venous sinus stenosis before placement of a VPS or LPS and that treatment options be discussed at a multidisciplinary conference. CSF diversion should be utilized cautiously due to the high cost and morbidity associated with revision surgeries. Based on our comprehensive literature review, Fig. 6 displays a potential management algorithm regarding the use of various surgical options for patients with medically refractory $\mathrm{IIH}$.

\section{Conclusions}

VSS for IIH has shown good outcomes for symptomatic improvement related to headache, vision, and tinnitus. $\mathrm{Pa}-$ tients with refractory IIH are medically complex, and the optimal surgical treatment should be determined in a multidisciplinary fashion. Additionally, we recommend that the patient continue on maximal medical management under the care of headache specialists with expertise in IIH, as other concomitant headache types are common in this patient population. ${ }^{23}$ While the current evidence appears promising regarding VSS in a subset of patients with IIH, future studies are required, with a focus on investigating the long-term outcomes for VSS, comparing its efficacy to CSF diversion and ONSF, and establishing the best candidates for stent placement, thus creating specific best-practice guidelines for the treatment of medically refractory IIH.

\section{References}

1. Ahmed RM, Wilkinson M, Parker GD, Thurtell MJ, Macdonald J, McCluskey PJ, et al: Transverse sinus stenting for idiopathic intracranial hypertension: a review of 52 patients and of model predictions. AJNR Am J Neuroradiol 32:1408-1414, 2011

2. Ahmed RM, Zmudzki F, Parker GD, Owler BK, Halmagyi GM: Transverse sinus stenting for pseudotumor cerebri: a cost comparison with CSF shunting. AJNR Am J Neuroradiol 35:952-958, 2014

3. Albuquerque FC, Dashti SR, Hu YC, Newman CB, Teleb M, McDougall CG, et al: Intracranial venous sinus stenting for benign intracranial hypertension: clinical indications, technique, and preliminary results. World Neurosurg 75:592595, 648-652, 2011

4. Arac A, Lee M, Steinberg GK, Marcellus M, Marks MP: Efficacy of endovascular stenting in dural venous sinus stenosis for the treatment of idiopathic intracranial hypertension. Neurosurg Focus 27(5):E14, 2009

5. Asif H, Craven CL, Siddiqui AH, Shah SN, Matloob $\mathrm{SA}$, Thorne L, et al: Idiopathic intracranial hypertension: 120-day clinical, radiological, and manometric outcomes after stent insertion into the dural venous sinus. J Neurosurg [epub ahead of print October 6, 2017. DOI: 10.3171/2017.4.JNS162871]

6. Banta JT, Farris BK: Pseudotumor cerebri and optic nerve sheath decompression. Ophthalmology 107:1907-1912, 2000

7. Bussière M, Falero R, Nicolle D, Proulx A, Patel V, Pelz D: Unilateral transverse sinus stenting of patients with idiopathic intracranial hypertension. AJNR Am J Neuroradiol 31:645-650, 2010

8. Dinkin MJ, Patsalides A: Venous sinus stenting for idiopathic intracranial hypertension: where are we now? Neurol Clin 35:59-81, 2017 
9. Donnet A, Metellus P, Levrier O, Mekkaoui C, Fuentes S, Dufour H, et al: Endovascular treatment of idiopathic intracranial hypertension: clinical and radiologic outcome of 10 consecutive patients. Neurology 70:641-647, 2008

10. Ducruet AF, Crowley RW, McDougall CG, Albuquerque FC: Long-term patency of venous sinus stents for idiopathic intracranial hypertension. J Neurointerv Surg 6:238-242, 2014

11. Elder BD, Goodwin CR, Kosztowski TA, Radvany MG, Gailloud P, Moghekar A, et al: Venous sinus stenting is a valuable treatment for fulminant idiopathic intracranial hypertension. J Clin Neurosci 22:685-689, 2015

12. Farb RI, Vanek I, Scott JN, Mikulis DJ, Willinsky RA, Tomlinson G, et al: Idiopathic intracranial hypertension: the prevalence and morphology of sinovenous stenosis. Neurology 60:1418-1424, 2003

13. Feldon SE: Visual outcomes comparing surgical techniques for management of severe idiopathic intracranial hypertension. Neurosurg Focus 23(5):E6, 2007

14. Fields JD, Javedani PP, Falardeau J, Nesbit GM, Dogan A, Helseth EK, et al: Dural venous sinus angioplasty and stenting for the treatment of idiopathic intracranial hypertension. J Neurointerv Surg 5:62-68, 2013

15. Friedman DI: The pseudotumor cerebri syndrome. Neurol Clin 32:363-396, 2014

16. Galgano MA, Deshaies EM: An update on the management of pseudotumor cerebri. Clin Neurol Neurosurg 115:252259,2013

17. Higgins JN, Cousins C, Owler BK, Sarkies N, Pickard JD: Idiopathic intracranial hypertension: 12 cases treated by venous sinus stenting. J Neurol Neurosurg Psychiatry 74:1662-1666, 2003

18. Higgins JN, Owler BK, Cousins C, Pickard JD: Venous sinus stenting for refractory benign intracranial hypertension. Lancet 359:228-230, 2002

19. Ibrahim MH, Zeid SHO, Abd Elbar A: Prevalence of venous sinus stenosis in pseudotumor cerebri (PTC) using digital subtraction angiography (DSA). Egypt J Radiol Nucl Med 45:519-522, 2014

20. Kanagalingam S, Subramanian PS: Cerebral venous sinus stenting for pseudotumor cerebri: a review. Saudi J Ophthalmol 29:3-8, 2015

21. Kumpe DA, Bennett JL, Seinfeld J, Pelak VS, Chawla A, Tierney M: Dural sinus stent placement for idiopathic intracranial hypertension. J Neurosurg 116:538-548, 2012

22. Markey KA, Mollan SP, Jensen RH, Sinclair AJ: Understanding idiopathic intracranial hypertension: mechanisms, management, and future directions. Lancet Neurol 15:78-91, 2016

23. Mathew NT, Ravishankar K, Sanin LC: Coexistence of migraine and idiopathic intracranial hypertension without papilledema. Neurology 46:1226-1230, 1996

24. Matloob SA, Toma AK, Thompson SD, Gan CL, Robertson $F$, Thorne L, et al: Effect of venous stenting on intracranial pressure in idiopathic intracranial hypertension. Acta Neurochir (Wien) 159:1429-1437, 2017

25. McGeeney BE, Friedman DI: Pseudotumor cerebri pathophysiology. Headache 54:445-458, 2014

26. McGirt MJ, Woodworth G, Thomas G, Miller N, Williams M, Rigamonti D: Cerebrospinal fluid shunt placement for pseudotumor cerebri-associated intractable headache: predictors of treatment response and an analysis of long-term outcomes. J Neurosurg 101:627-632, 2004

27. Mollan SP, Ali F, Hassan-Smith G, Botfield H, Friedman DI, Sinclair AJ: Evolving evidence in adult idiopathic intracranial hypertension: pathophysiology and management. J Neurol Neurosurg Psychiatry 87:982-992, 2016

28. Mukherjee N, Bhatti MT: Update on the surgical management of idiopathic intracranial hypertension. Curr Neurol Neurosci Rep 14:438, 2014
29. Ogungbo B, Roy D, Gholkar A, Mendelow AD: Endovascular stenting of the transverse sinus in a patient presenting with benign intracranial hypertension. Br J Neurosurg 17:565568,2003

30. Owler BK, Besser M: Extradural hematoma causing venous sinus obstruction and pseudotumor cerebri syndrome. Childs Nerv Syst 21:262-264, 2005

31. Owler BK, Parker G, Halmagyi GM, Dunne VG, Grinnell V, McDowell D, et al: Pseudotumor cerebri syndrome: venous sinus obstruction and its treatment with stent placement. J Neurosurg 98:1045-1055, 2003

32. Paquet C, Poupardin M, Boissonnot M, Neau JP, Drouineau J: Efficacy of unilateral stenting in idiopathic intracranial hypertension with bilateral venous sinus stenosis: a case report. Eur Neurol 60:47-48, 2008

33. Portelli M, Papageorgiou PN: An update on idiopathic intracranial hypertension. Acta Neurochir (Wien) 159:491-499, 2017

34. Puffer RC, Mustafa W, Lanzino G: Venous sinus stenting for idiopathic intracranial hypertension: a review of the literature. J Neurointerv Surg 5:483-486, 2013

35. Radvany MG, Solomon D, Nijjar S, Subramanian PS, Miller NR, Rigamonti D, et al: Visual and neurological outcomes following endovascular stenting for pseudotumor cerebri associated with transverse sinus stenosis. J Neuroophthalmol 33:117-122, 2013

36. Rajpal S, Niemann DB, Turk AS: Transverse venous sinus stent placement as treatment for benign intracranial hypertension in a young male: case report and review of the literature. J Neurosurg 102 (3 Suppl):342-346, 2005

37. Satti SR Sr, Leishangthem L, Chaudry MI: Meta-analysis of CSF diversion procedures and dural venous sinus stenting in the setting of medically refractory idiopathic intracranial hypertension. AJNR Am J Neuroradiol 36:1899-1904, 2015

38. Sinclair AJ, Kuruvath S, Sen D, Nightingale PG, Burdon MA, Flint G: Is cerebrospinal fluid shunting in idiopathic intracranial hypertension worthwhile? A 10-year review. Cephalalgia 31:1627-1633, 2011

39. Spoor TC, McHenry JG: Long-term effectiveness of optic nerve sheath decompression for pseudotumor cerebri. Arch Ophthalmol 111:632-635, 1993

40. Starke RM, Wang T, Ding D, Durst CR, Crowley RW, Chalouhi N, et al: Endovascular treatment of venous sinus stenosis in idiopathic intracranial hypertension: complications, neurological outcomes, and radiographic results. Sci World J 2015:140408, 2015

41. Teleb MS, Rekate H, Chung S, Albuquerque FC: Pseudotumor cerebri presenting with ataxia and hyper-reflexia in a non-obese woman treated with sinus stenting. J Neurointerv Surg 4:e22, 2012

42. ten Hove MW, Friedman DI, Patel AD, Irrcher I, Wall M, McDermott MP: Safety and tolerability of acetazolamide in the idiopathic intracranial hypertension treatment trial. J Neuroophthalmol 36:13-19, 2016

43. Wall M: Idiopathic intracranial hypertension. Neurol Clin 28:593-617, 2010

44. Wall M: Update on idiopathic intracranial hypertension. Neurol Clin 35:45-57, 2017

45. Zheng H, Zhou M, Zhao B, Zhou D, He L: Pseudotumor cerebri syndrome and giant arachnoid granulation: treatment with venous sinus stenting. J Vasc Interv Radiol 21:927-929, 2010

\section{Disclosures}

Dr. Prestigiacomo reports a consultant relationship with Cerenovus, Edge Therapeutics, and Thermopeutix. 


\section{Author Contributions}

Conception and design: Prestigiacomo, Giridharan, Patel, Nouri. Acquisition of data: Giridharan, Patel, Ojugbeli. Analysis and interpretation of data: Prestigiacomo, Giridharan, Patel, Ojugbeli. Drafting the article: Giridharan, Patel, Ojugbeli. Critically revising the article: Prestigiacomo, Giridharan, Patel, Nouri, Shirani, Grossman, Cheng, Zuccarello. Reviewed submitted version of manuscript: all authors. Approved the final version of the manu- script on behalf of all authors: Prestigiacomo. Administrative/ technical/material support: Patel, Nouri. Study supervision: Prestigiacomo, Giridharan, Patel.

\section{Correspondence}

Charles J. Prestigiacomo: University of Cincinnati College of Medicine, Cincinnati, OH. presticj@ucmail.uc.edu. 